\title{
EL DESARROLLO DE LOS PROCESOS UNIVERSITARIOS DESDE LA GESTIÓN ACADÉMICA EN RESPUESTA A SU RESPONSABILIDAD SOCIAL
}

Silvia Cruz Baranda, Dra. C. Universidad de Oriente, Cuba silvia@uo.edu.cu

\author{
Daniel Fuentes Almaguer, Dr. C. \\ Universidad de Oriente, Cuba \\ daniel.fuentes@uo.edu.cu
}

\author{
Nirma Pérez Pelipiche, Dra. C \\ Universidad de Oriente, Cuba \\ nirmapp@uo.edu.cu
}

Palabras claves: gestión académica, procesos universitarios, responsabilidad social

Keywords: academic management, university processes, social responsibility

Recibido: 15 de Septiembre de 2017

Aceptado: 09 de Octubre de 2017

\section{RESUMEN}

Cada vez son más numerosos los proyectos de investigación conjuntos entre universidades y empresas e instituciones, porque es la forma de renovar la educación tanto de pregrado como de posgrado. Al tiempo que permiten que los resultados de las investigaciones se introduzcan efectivamente en los sectores que lo demandan, de este modo la universidad cumple sus funciones extensionistas, basadas en la colaboración interinstitucional. Se incrementa la comprensión científica de los procesos sociales y tecnológicos, la capacidad de innovación y la necesidad del aprendizaje continuo. En el trabajo se exponen los resultados parciales de un proyecto de investigación, a partir del trabajo coordinado de la Vicerrectoría Docente y la Vicerrectoría de Investigaciones y Posgrado, para trabajar desde el curso 15-16 los procesos universitarios con una mirada integradora desde la gestión académica universitaria en el territorio en respuesta a su responsabilidad social.

\section{ABSTRACT}

There is a growing number of joint research projects among universities, companies and institutions because it is the way to renew education, both undergraduate and postgraduate, while research results are effectively introduced in the demanding sectors, the ones the university fulfills the extensionist functions with, based on inter-institutional collaboration, increasing scientific understanding of social and technological processes, the capacity for innovation and the need for continuous learning. This paper presents the partial results of a research project based on the coordinated work of the Vice-Rector's Office and the ViceRectory of Research and Post-graduate studies to work from the 2015 -2016 academic years on university processes with an integrated approach considering the university academic management in response to its social responsibility. 


\section{INTRODUCCIÓN}

Se asume la universidad como un sistema de procesos, en el cual concurren los procesos esenciales de formación, investigación y extensión universitaria, se hace imprescindible que los mismos asuman los desafíos de la época actual en términos de educación continua y respuestas apropiadas al desarrollo social y económico. En la actualidad resulta inaplazable que las universidades demuestren la idoneidad de sus procesos a través de la pertinencia y el impacto de sus resultados. Según Giustiet lo que falta en América Latina es la implementación práctica de una concepción de profesor-investigador (o investigadorprofesor) en la cual la docencia, la investigación y la extensión se interrelacionen y completen de manera armónica para dar lugar a un nuevo perfil de profesional docente universitario. Giustiet. (2013). Las posibilidades de integración de las universidades con los sectores productivos y de servicios a través de los resultados que se ofrecen desde la investigación, el posgrado y la extensión universitaria, así como la integración de los estudiantes a las prácticas laborales e investigativas, se constituye en una tarea latente de las universidades, siendo la manera de garantizar el retorno permanente de las acciones sociales emprendidas hacia el mejoramiento de la formación académica y profesional de la comunidad universitaria, al tiempo que se promueve su responsabilidad social.

La responsabilidad social universitaria es una política de gestión de la calidad ética de la universidad, que busca alinear sus cuatro procesos (gestión, docencia, investigación, extensión) con la misión universitaria, sus valores y compromiso social, mediante el logro de la congruencia institucional, la transparencia y la participación dialógica de toda la comunidad universitaria (autoridades, estudiantes, docentes, administrativos) con los múltiples actores sociales interesados en el buen desempeño universitario y necesitados de él, para la transformación efectiva de la sociedad hacia la solución de sus problemas de exclusión, inequidad, y sostenibilidad. Vallaeys (2007). De ahí, que la responsabilidad social universitaria se presenta como un desafío a las universidades, dado que esto implica que los centros de educación superior se comprometen no sólo a formar buenos profesionales, sino también personas sensibles a los problemas de los demás, comprometidas con el desarrollo de su país y la inclusión social de los más vulnerables, personas entusiastas y creativas en la articulación de su profesión con la promoción del desarrollo participativo de su comunidad.

Por eso, la formación del profesional en el pregrado, el posgrado, la investigación científica y la extensión universitaria, deben basarse en la construcción de un sistema educativo absolutamente incluyente, donde todos los ciudadanos tienen el derecho constitucional de estudiar gratuitamente a lo largo de toda su vida. De la calidad de ese sistema depende, en gran medida, la existencia de una capacidad cultural, de una racionalidad extendida, que permite a la sociedad enfrentar desafíos productivos, políticos y disponer de un nivel razonable de capacidades profesionales y de investigación que permitan formular y responder preguntas relevantes en ámbitos tan disímiles como economía, salud, cultura, educación, agricultura, turismo, sociedad, medio ambiente, prevención contra riesgos y desastres, entre otros. Esa capacidad de indagación es determinante para poder crear e impulsar agendas de investigación que respondan a los problemas más importantes del país. Núñez (2010). 
El interés de que a través del pregrado y el posgrado los profesionales se erijan como verdaderos transformadores de los procesos sociales, productivos y de servicios en los que se involucran, potencia la necesidad de integración con la investigación y la extensión, como procesos universitarios, a fin de propender a la innovación social. Esta ultima puede ser definida como el desarrollo e implementación de nuevas ideas (productos, servicios y modelos) para satisfacer las necesidades sociales, crear nuevas relaciones sociales y ofrecer mejores resultados. Sirve de respuesta a las demandas sociales que afectan al proceso de interacción social, dirigiéndose a mejorar el bienestar humano. Rodríguez (2008).

Las Innovaciones sociales son aquellas que no sólo son buenas para la sociedad, sino que mejoran la capacidad de actuación de las personas. Se basan en la creatividad de los ciudadanos, las organizaciones de la sociedad civil, las comunidades locales o las empresas. Son una oportunidad tanto para el sector público como para los mercados, de modo que los productos y servicios satisfacen las aspiraciones individuales y colectivas. Engel, (199). De ahí, que la universidad debe generar los procesos de transformación en el entorno, en coordinación con todas las instituciones y entidades económicas, políticas y sociales del mismo. Por esto, se debe formar a los profesionales para participar activamente en los procesos de la comunidad, con protagonismo, compromiso, laboriosidad, productividad, haciendo uso de la crítica constructiva, de la reflexión, la creatividad y la innovación.

En los reiterados análisis de los resultados alcanzados por la Universidad de Oriente, producto de la gestión de sus procesos, se han revelado insuficiencias en relación con el vínculo entre la universidad y las entidades de producción y servicios del territorio, las que tienen incidencia en la calidad de los graduados, en la calidad del posgrado, en la concertación de proyectos de investigación conjuntos y proyectos socio-comunitarios, por ende, en la capacidad de la universidad para resolver problemas del desarrollo socioeconómico del territorio. Algunas de estas insuficiencias son:

$\checkmark \quad$ Aún no se alcanzan los niveles deseados de participación de estudiantes en las investigaciones científicas relacionadas con problemas territoriales que forman parte de los bancos de problemas de las empresas e instituciones.

$\checkmark \quad$ Limitaciones en las solicitudes de posgrado que hacen las EP\&S que no reflejan las exigencias de desempeños orientados al desarrollo profesional creciente, según lo demandan los avances de la ciencia y la tecnología y el perfeccionamiento de las organizaciones en que trabajan.

Entre la causa fundamental de esas insuficiencias aflora la manera en que se han venido gestionando los procesos universitarios, donde no siempre se hace énfasis en el vínculo efectivo con las entidades de producción y servicios y la universidad. Es objetivo de este trabajo presentar un proyecto que integre la formación del profesional de pregrado, de posgrado, la ciencia e innovación tecnológica y la extensión universitaria, con las entidades de producción y servicios del territorio, con vistas a su contribución al desarrollo socio económico del territorio. 


\section{DESARROLLO}

El objetivo general del proyecto que es: elevar el impacto de la Universidad de Oriente en el territorio oriental del país, a través de una estrategia de gestión académica integrada de los procesos de formación del profesional de pregrado, de posgrado, de ciencia e innovación tecnológica y de extensión universitaria, en su vínculo con las entidades de producción y servicios del territorio, utilizando el método de estudio de casos.

Para dar cumplimiento a este objetivo se valora que el vínculo de las universidades con sus contextos favorece en gran medida que la gestión del conocimiento irradie a todos los procesos económicos y sociales, y que la sociedad toda se convierta en agente del conocimiento.

La interacción entre estudiantes universitarios, profesores, profesionales y actores sociales implica el crecimiento y desarrollo científico y académico, lo que significa conformar y dinamizar comunidades reflexivas en interrelación sistémica y con sistematicidad en interacción e interrelación para el intercambio de ideas y la comunicación a favor del desarrollo profesional y social. El conocimiento y la innovación que las universidades producen y difunden son fundamentales en los grandes propósitos del desarrollo social, la inclusión y la competitividad de los sectores productivos, sobre todo en el contexto de una economía globalizada e interconectada. Alarcón (2016).

Asegurar la calidad de los procesos universitarios tiene como objetivo esencial la obtención de resultados útiles a la sociedad, susceptibles de ser generalizados con el fundamento básico de la satisfacción de las necesidades sociales. Se requiere, por tanto, que la comunidad universitaria sea portadora de una cultura creativa e innovadora, con capacidad para generar conocimiento sobre su realidad y su entorno, con el compromiso y la responsabilidad compartida de asumir cambios hacia el mejoramiento continuo. Estos propósitos no se alcanzan de forma espontánea, dependen de la forma en que se gestionan los procesos universitarios.

El sistema de gestión debe llevar a la universidad al cumplimiento de los objetivos propuestos con una utilización eficiente de los recursos para brindar los impactos sociales, económicos y científicos y satisfacción de las demandas sociales, es decir, lograr los objetivos de la universidad, a través del aprovechamiento del capital intelectual, los bienes y recursos. Muestra de que el trabajo científico y de desarrollo tecnológico es creativo por su esencia humana, y en cuanto a los procesos de cambio que genera, es innovador a decir de Hernández (2006). Se impone la necesidad de perfeccionar los procesos universitarios, desde su propia gestión, dirigida a incrementar la confianza y motivación de sus actores. Es lo que Benno Sander denomina "una construcción integradora de administración de la educación, preocupada por satisfacer, simultáneamente, las necesidades individuales y las exigencias colectivas de la ciudadanía en términos de utilización de los servicios educativos." Sander (1996). 
En consecuencia, la gestión de los procesos formativos universitarios en la educación superior debe ampliar su visión e identificar su dimensión académica para ubicar y definir el compromiso y la participación de sus actores en todos los procesos universitarios tendientes al logro de sus propósitos, como institución educativa socialmente comprometida. Darle mayor significación a la dimensión académica de la gestión universitaria, implica generar procesos de transformación que impacten el vínculo existente entre los centros de educación superior y el entorno social.

En este sentido, Sander (1996) propone repensar la gestión educativa, acorde a la propia realidad y las influencias de reciprocidad con su entorno, lo cual exige un tratamiento singular. Se considera que la gestión universitaria posee en sí misma una complejidad, la cual incluye subjetividades de las personas, la realidad institucional y fundamentalmente el entorno, destacándose la importancia de que la gestión incluya un saber sobre la realidad en que se interviene, lo cual define la intencionalidad pedagógica de la misma.

Las características singulares de los procesos universitarios, obligan que su gestión descanse en una gestión académica, en la que participan todos los sujetos involucrados: profesores, estudiantes, directivos y todos cuantos, contraigan algún compromiso con las metas trazadas institucionalmente. A esto, se une la necesidad de que la gestión académica trascienda los ámbitos universitarios y se proyecte a las empresas, instituciones, a la comunidad, con una visión transformadora, de compromiso y responsabilidad social.

La gestión académica, es entendida por S. Cruz y B. García (2013), como el conjunto de acciones docentes e investigativas que se despliegan a favor del cumplimiento de los propósitos más trascendentes de la institución, con una participación activa de todos los sujetos implicados y haciendo énfasis en el aprendizaje de contenidos, formas y métodos de gestión que movilizan esfuerzos por la obtención de resultados pertinentes y de impacto.

Siendo así, la gestión académica de los procesos de formación (pregrado y posgrado), de investigación y de extensión, implica aprovechar las prácticas laborales - investigativas, los programas de maestrías, convocar a los innovadores, evaluar sus proyectos, seleccionarlos, reconocerlos públicamente, difundir sus logros destacando la novedad, sistematizarlos y mediar en los procesos que conduzcan a su inclusión como parte de la política pública. Se trata, en última instancia, de interceder para crear conectividad entre la universidad y las entidades de producción y servicios del territorio, de modo que se logre abrir la posibilidad de una acción social conjunta entre los actores universitarios y los agentes sociales.

Lo anterior, genera la necesidad de formular planes de acción que tengan en cuenta los recursos, capacidades y la dinámica del contexto, tratando de establecer tareas específicas derivadas de objetivos generales que contemplen las especificidades propias de los procesos y operacionalizarlas de aquellos factores o variables más influyentes para orientar el logro de resultados eficientes, efectivos y eficaces. 
En los medios académicos existe consenso al reconocer que las universidades tienen formas fundamentales de intervenir activamente en los sectores de producción, de servicios y de participación comunitaria y con ello asumir la responsabilidad social: generando nuevos conocimientos científicos, tanto básicos como aplicados, a través de sus propios grupos de investigación con el apoyo del sector público y privado; dando formación con alta calidad a los profesionales y a la sociedad en general, para satisfacer las demandas de estos sectores y cristalizando los avances de la investigación, creando una red de empresas industriales y de nuevos negocios.

También se discute acerca de las dificultades que se ponen de manifiesto desde el ámbito empresarial, el académico y el de los centros tecnológicos y que surgen por las diferencias en múltiples aspectos: en los objetivos de las organizaciones implicadas, en los motivos para colaborar, en los estilos de gestión, en las nociones de tiempo, en las nociones de confidencialidad. Por eso, la importancia y la significación que tiene la gestión académica, entendida como un proceso que convoca, aglutina y promueve la participación y colaboración, ya que basa sus métodos en estrategias pedagógicas basadas en la sensibilización y sistematización para identificar, capturar y documentar aprendizajes y experiencias de proyectos y programas.

De ahí, su carácter eminentemente pedagógico, que encuentra sus fundamentos conceptuales en la pedagogía social, que es la ciencia capaz de analizar las condiciones sociales de la cultura y las condiciones culturales de la vida social, y el hombre, según Pérez, (2003); y que se ocupa de "la educación en sus relaciones con la sociedad, es decir, la acción de los grupos sociales en la formación del hombre y la influencia de la educación en la sociedad humana". Luzuriaga (1968). Desde este punto de vista la gestión académica de los procesos universitarios debe propender a que los actores universitarios a profundizar y ampliar en el espectro de herramientas y conocimientos para ponerlos en función de su accionar diario con las comunidades y en función de la gobernabilidad, con énfasis en la multiplicación de las acciones prácticas y los aprendizajes, será posible que los procesos no desaparezcan y que evolucionen de acuerdo con las necesidades y posibilidades reales de cada lugar. Mirabal (2006).

En las condiciones actuales de la Universidad de Oriente integrada para atender 55 carreras desde 13 facultades y con más de 250 centros categorizados como unidades docentes (centros docentes, de servicios y empresas) genera las condiciones necesarias para atender con un enfoque más integral los procesos formativos universitarios y alcanzar niveles superiores de gestión. Se trata la gestión universitaria, con el propósito de involucrar a todos los sujetos en sus procesos, y que propicie la participación y colaboración consciente, la disposición e implicación en las acciones institucionales y a la incorporación de todos al mejoramiento y cambio, a favor del cumplimiento de las metas y objetivos trazados, lo que se traduce en una gestión incluyente, una ruptura entre las formas tradicionales de administrar y concebir el proceso de gestión vinculado al desarrollo individual y colectivo, a partir de comprenderla propia naturaleza del conocimiento. 
Tareas a desarrollar para dar cumplimiento al objetivo general del proyecto.

1) Evaluar los vínculos de la UO con las entidades laborales de producción o servicios del territorio con la participación de los representantes de dichas entidades.

2) Sistematizar el contenido conceptual de la relación universidad -sociedad, contextualizado a las particularidades de la UO y el territorio con el que interactúa.

3) Identificar las entidades laborales de producción o servicios que participarán en el estudio de casos.

4) Caracterizar el estado actual de la relación universidad - sociedad en las entidades laborales de producción o servicios seleccionadas.

5) Organizar curso de posgrado que integre la gestión universitaria de la relación universidad - sociedad, contextualizado a las particularidades actuales de la UO.

6) Diseñar las estrategias a desarrollar en cada una de las entidades laborales de producción o servicios e implementarlas en su $1^{\text {a }}$ etapa.

7) Evaluar los resultados de la implementación de las estrategias en su $1^{\text {a }}$ etapa.

\section{Resultados y Discusión}

Valoración de los resultados de la tabulación de la encuesta aplicada a directivos y profesionales de las Unidades Docentes (UD) y Entidades Laborarles de Base (ELB)

Fueron encuestados un total de 31 directivos de diferentes UD o ELB en las mismas se tuvo como resultados por pregunta cómo sigue. De los encuestados 26 que representan el $83.87 \%$ marcan como buena la contribución del postgrado y las prácticas laborales investigativas que se realizan por parte de la Universidad de Oriente al mejoramiento del desempeño de los profesionales que dirigen; 4 refieren es regular y uno la califica de deficiente agregando que:

$\checkmark \quad$ Existe poco seguimiento al graduado universitario a partir que se considera que ya cuenta con todas las capacidades profesionales para su desarrollo

$\checkmark \quad$ Las ofertas de los postgrados no satisfacen plenamente las necesidades de las prácticas laborales investigativas

Los encuestados identifican como aspectos positivos de la contribución del postgrado y las prácticas laborales las siguientes:

$\checkmark$ Mejor dominio de competencias profesionales

$\checkmark$ Mayor actualización científica

$\checkmark$ Mayor compromiso profesional

$\checkmark$ Mejores relaciones interpersonales

$\checkmark$ Mayor actualización profesional

$\checkmark$ Promoción a funciones de mayor responsabilidad profesional

$\checkmark$ Promoción a cargos de dirección

$\checkmark$ Mayor integración a equipos multidisciplinarios

$\checkmark$ Obtención de premios o reconocimientos 
En cuanto a la pregunta 4 de la encuesta sobre identificar algunas de las contribuciones que el directivo considera que las prácticas laborales investigativas y las actividades de postgrado han hecho a la institución consideran:

$\checkmark \quad$ Con un $70.6 \%$ la elevación del nivel profesional de los trabajadores

$\checkmark$ Con un $61.2 \%$ mayor participación de trabajadores en eventos de carácter profesional.

$\checkmark \quad 48.3 \%$ solución a problemas técnicos y el mejoramiento de las relaciones interpersonales

$\checkmark$ Con $45.16 \%$ mayores niveles de satisfacción con los servicios prestados y un $41.9 \%$ indican mejoría en los indicadores en el uso de los recursos materiales

El $75 \%$ de los encuestados refieren la existencia de proyectos I+D+i en la entidad. Emiten como consideraciones importantes la necesidad de:

$\checkmark \quad$ Involucrar en las tareas a los profesionales más capaces

$\checkmark$ Convocar a reuniones periódicas a los participantes en los proyectos

$\checkmark$ Debatir colectivamente los resultados de las tareas de los proyectos

$\checkmark$ Estimular la colaboración entre los participantes en los proyectos

$\checkmark$ Prever la publicación y difusión de los resultados de los proyectos

$\checkmark$ Controlar la aplicación y generalización de los resultados de los proyectos

$\checkmark$ Controlar el uso de los recursos asignados a los proyectos

El $78 \%$ de los encuestados consideran la participación de profesores y/o estudiantes de la UO beneficiosa para el avance de las tareas de los proyectos I+D+i y proponen:

$\checkmark$ Ampliar y profundizan en los conocimientos requeridos a los temas de investigación

$\checkmark$ Aprovechar los resultados de investigaciones similares desarrolladas anteriormente en la UO

$\checkmark \quad$ Utilizar medios y recursos tecnológicos con que cuenta la UO

$\checkmark$ Aprovechar el caudal de conocimientos de la UO en torno a la gestión de proyectos

$\checkmark$ Incrementar el número de investigadores, incluyendo a profesores y estudiantes en los equipos de investigación

El $80.64 \%$ de los directivos encuestados refieren haber tenido estudiantes de práctica laboral en su centro en los últimos años valorando el $70.96 \%$ haberse realizado éstas con calidad el desempeño de los estudiantes y un $9 \%$ califican de regular su desempeño por:

$\checkmark \quad$ No tener preparación suficiente para las tareas profesionales que se les asignan

$\checkmark \quad$ No tener responsabilidad con el cumplimiento de sus funciones profesionales

$\checkmark$ No mostrar suficiente interés por el desarrollo profesional. 


\section{Resultados parciales}

Cada tarea de investigación propuesta ha tenido un aseguramiento desde las reuniones del proyecto y se alcanzan resultados loables parciales a partir de la aplicación del proyecto, el que ha realizado las siguientes actividades:

1) Realización de 10 talleres científicos-metodológicos: universidad - sociedad que han permitido el intercambio con representantes de los Organismos de la Administración Central del Estado en el territorio (OACE), mejorando las relaciones de trabajo de estos con las facultades, carreras y áreas de la universidad.

2) Se tomó una muestra de 25 unidades docentes que atienden diferentes carreras de la universidad, se visitaron y se aplicó un diagnóstico que permitió identificar las entidades laborales de producción o servicios que participarán en el estudio de casos.

3) Se han desarrollado 10 actividades metodológicas de preparación de los jefes de carreras y jefes de departamento docente para la atención a la práctica laboral de los estudiantes.

4) Se planificaron las prácticas laborales, lo que permitió realizar convenios de trabajo con las entidades laborales a partir del criterio de satisfacción de los estudiantes y los representantes de las entidades laborales.

5) Se realizó por parte de las entidades laborales el diagnóstico de necesidades de superación permitiendo una mejor atención al postgrado desde una planificación de los cursos por parte de las carreras y las facultades.

Se aprecia un mayor intercambio y participación de las entidades laborales para mejorar la actividad investigativa de los estudiantes.

\section{CONCLUSIÓN}

De forma parcial se valora que el impacto de la Universidad de Oriente en el territorio oriental del país a través de una estrategia de gestión académica integrada de los procesos de formación del profesional, de posgrado, de ciencia e innovación tecnológica y de extensión universitaria, en su vínculo con las entidades de producción y servicios del territorio, utilizando el método de estudio de casos ha permitido mejorar paulatinamente los intercambios con los representantes de los OACE y las entidades laborales para de conjunto perfeccionar la formación del profesional.

\section{BIBLIOGRAFÍA}

1. Alarcón, R (2016): Universidad innovadora por un desarrollo sostenible. Conferencia inaugural 10mo. Congreso Internacional Universidad 2016, La Habana, Cuba.

2. Arias Guevara María de los Ángeles y Ciro Miguel Labrada Silva (2008): Debate actual sobre el desarrollo. Ruptura con la visión tradicional. En Estudios sobre desarrollo local, innovación social y género. Editorial Academia. La Habana, Cuba. 
3. Bayona Sáez, Cristina y Raquel González Eransus (2010): La transferencia de conocimiento en la Universidad Pública de Navarra. Una visión desde la empresa y desde el ámbito universitario. Sección de Publicaciones Universidad Pública de Navarra.

4. Cruz Baranda, Silvia y María B. García Quiala (2013): La gestión académica del posgrado y los conceptos que conducen a la pertinencia y al impacto. Revista Congreso Universidad, Vol. 2, No. 1 (2013). En: http://www.congresouniversidad.cu/revista. [consulta: 12.02.2014].

5. Engel, Paul (1997): La organización social de la innovación. Enfocando en/sobre la interacción de los agentes involucrados, Santiago de Chile, KIT Press, Royal Tropical Institute.

6. Giustit Pachano, Graziela y Roberto Valdés Puentes (2005): "La Universidad y la distribución social del conocimiento. ¿Sus profesores están preparados?”. Universidad de Granada. En: http://prensa.ugr.es/prensa/dialogo/biblioteca/mass_media_universidad/. [consulta: 17.08.2013]

7. Hernández, J.L. (2006): "La ciencia y la tecnología como factores de desarrollo. El espacio local”. En: Desarrollo local en Cuba. Comp: Ada Guzón Camporredondo, Editorial Academia, Cuba.

8. Ibarra Colado, Eduardo (2001): La universidad en México hoy, gubernamentalidad y modernización. México. ANUIES. 